DOI 10.1007/s10986-021-09531-y

Lithuanian Mathematical Journal, Vol. 61, No. 3, July, 2021, pp. 413-423

\title{
Distinguishing $L$-functions by joint universality
}

\author{
Jörn Steuding \\ Department of Mathematics, Würzburg University, Emil Fischer-Str. 40, 97074 Würzburg, Germany \\ (e-mail: steuding@mathematik.uni-wuerzburg.de) \\ Dedicated to the Memory of Jonas Kubilius at the Occasion of his 100th Birthday
}

Received January 17, 2021; revised March 24, 2021

\begin{abstract}
In this note, we present results for distinguishing $L$-functions by their multisets of zeros and unique factorizations in an axiomatic setting; our tools stem from universality theory.
\end{abstract}

MSC: 11M06, 30D35

Keywords: $L$-functions, universality, zero-distribution

\section{Motivation and statement of the main results}

Zeta- and $L$-functions play a central role in analytic number theory. For example, they have been used by Erich Hecke [10] and Jonas Kubilius [15] for their studies of the ratios $a / b$ arising from representations of prime numbers $p \equiv 1(\bmod 4)$ as a sum of two squares $p=a^{2}+b^{2}$ with respect to uniform distribution (in short arcs). In a recent paper, Zeév Rudnick and Ezra Waxman [30] investigated fine scale statistics of these angles. In this short note, we are concerned with a rather different theme on $L$-functions.

There are several axiomatic settings for number-theoretically relevant zeta- and $L$-functions; the probably most investigated ones are the so-called Selberg class $\mathcal{S}$, introduced by Atle Selberg [31] in 1989, and the extended Selberg class $\mathcal{S}^{\sharp}$, respectively. It is an open problem to show that every element can be written as a unique product of irreducible elements, also called primitive $L$-functions. The existence of such a product representation is an immediate consequence of the Riemann-von Mangoldt formula counting the nontrivial zeros (resp., the notion of a degree within $\mathcal{S}^{\sharp}$ ), however, the uniqueness is unsettled. In fact, distinguishing $L$-functions by their zeros or, more generally, by their $a$-points is one possible approach. Using Hadamard's product theorem and estimates from Nevanlinna theory, the author [34] showed that if two elements of $\mathcal{S}$ share a complex value $a \neq 1$ counting multiplicities, then they are already identical (in fact, the statement in [34] is more general); this direction has been further developed in particular by Steven Gonek, Jaeho Haan, and Haseo $\mathrm{Ki}$ [9] and Bao Qin Li [21], to name only a few. Here we will not use Nevanlinna theory or any advanced tool from complex analysis (and therefore we may omit explaining the latter results in detail) but apply universality theorems.

Generalizing from the case of the well-known Riemann zeta-function $\zeta$, the zeros of any function $\mathcal{L} \in \mathcal{S}^{\sharp}$ are usually separated into the set of trivial zeros located at the poles of the gamma factors of the related functional equation and the set of all others, which are the so-called nontrivial zeros. The cardinality of both 
sets is measured by the degree of the function, denoted as $\operatorname{deg} \mathcal{L}$. The definition of this quantity and those of $\mathcal{S}$ and $\mathcal{S}^{\sharp}$ are given in Section 2. It turns out that the inequality

$$
\operatorname{deg} \mathcal{L}_{1}<\operatorname{deg} \mathcal{L}_{2}
$$

implies that there are many trivial and many nontrivial zeros of $\mathcal{L}_{2}$ that have a larger multiplicity than for $\mathcal{L}_{1}$; in the generic case, here we expect zeros $\rho$ satisfying $\mathcal{L}_{2}(\rho)=0 \neq \mathcal{L}_{1}(\rho)$; however, as the case $\mathcal{L}_{2}=\zeta^{2}$ shows, in general, the multiplicities have to be taken into account. Of course, the multiplicity may also be equal to zero, in which case the function does not vanish (as $\mathcal{L}_{1}$ at $s=\rho$ above).

Therefore, in our first result, we will consider the most interesting case of (almost) equal degrees.

Theorem 1. For $j=1,2$, let $\mathcal{L}_{j}$ be entire elements of $\mathcal{S}^{\sharp}$ and denote by $\mathcal{Z}_{j}$ the multiset of their zeros.

(i) Suppose that

$$
\mathcal{Z}_{1} \subset \mathcal{Z}_{2}
$$

and

$$
\operatorname{deg} \mathcal{L}_{1}+1>\operatorname{deg} \mathcal{L}_{2}
$$

If the constant term $a_{1}(1)$ in the Dirichlet series representation of $\mathcal{L}_{1}$ does not vanish, then there exists a Dirichlet series $\mathcal{D}$, absolutely convergent in the whole complex plane, such that

$$
\mathcal{L}_{2}=\mathcal{L}_{1} \cdot \mathcal{D}
$$

(ii) If $\mathcal{L}_{1}$ and $\mathcal{L}_{2}$ are jointly universal and $a_{1}(1) \neq 0$, then at least one of relations (1.1) and (1.2) cannot hold.

Note that in the multisets the zeros are listed according to their multiplicities. This shows that (1.1) and (1.2) imply $0 \leqslant \operatorname{deg} \mathcal{L}_{2}-\operatorname{deg} \mathcal{L}_{1}<1$. It has been conjectured that the degree is always a nonnegative integer (cf. [13, Sect. 9]). What is meant with the notion of joint universality is explained in Section 4. In our second result, we provide an application of this property on unique factorization.

Theorem 2. Joint universality for primitive L-functions implies unique factorization in $\mathcal{S}$ (resp., $\mathcal{S}^{\sharp}$ ).

In the subsequent five sections, we give the proofs of these two theorems (plus necessary information about the extended Selberg class and the phenomenon of universality). In the final section, we briefly discuss possible extensions of our reasoning and the research of others.

\section{The (extended) Selberg class}

We write the complex variable as $s=\sigma+$ it with the imaginary unit $\mathrm{i}=\sqrt{-1}$ in the upper half-plane.

We begin with the definition of the Selberg class. This class $\mathcal{S}$ consists of the functions $\mathcal{L}$ satisfying the following axioms:

(i) Dirichlet series: There is a representation of $\mathcal{L}$ as an absolutely convergent Dirichlet series for $\sigma>1$ :

$$
\mathcal{L}(s):=\sum_{n \geqslant 1} a(n) n^{-s}
$$

(ii) Analytic continuation: There exists an integer $k \geqslant 0$ such that $(s-1)^{k} \mathcal{L}(s)$ is an entire function of finite order. 
(iii) Functional equation: For every $s$,

$$
\mathfrak{L}(s)=\omega \overline{\mathfrak{L}(1-\bar{s})},
$$

where

$$
\mathfrak{L}(s):=\mathcal{L}(s) Q^{s} \prod_{1 \leqslant j \leqslant f} \Gamma\left(\lambda_{j} s+\mu_{j}\right)
$$

with positive real numbers $Q, \lambda_{j}$ and complex numbers $\mu_{j}, \omega$ satisfying $\Re \mu_{j} \geqslant 0$ and $|\omega|=1$.

(iv) Ramanujan hypothesis: For every $\epsilon>0$ and every positive integer $n$, we have $a(n) \ll n^{\epsilon}$, where the implicit constant may depend on $\epsilon$.

(v) Euler product: There is a product representation

$$
\mathcal{L}(s)=\prod_{p} \mathcal{L}_{p}(s)
$$

where the product is taken over all prime numbers $p$, and

$$
\mathcal{L}_{p}(s):=\exp \left(\sum_{k \geqslant 1} b\left(p^{k}\right) p^{-k s}\right)
$$

with suitable coefficients $b\left(p^{k}\right)$ satisfying $b\left(p^{k}\right) \ll p^{k \theta}$ for some $\theta<1 / 2$.

The larger set $\mathcal{S}^{\sharp}$ consisting of those functions $\mathcal{L}$ satisfying axioms (i)-(iii) is called the extended Selberg class. Usually, only the elements in $\mathcal{S}$ are called $L$-functions (originating from the naming of Dirichlet's " $L$ functions" to residue class characters); it is the Euler product that makes those so important for number theory!

The degree of an element $\mathcal{L} \in \mathcal{S}^{\sharp}$ is defined via the functional equation as

$$
\operatorname{deg} \mathcal{L}=2 \sum_{1 \leqslant j \leqslant f} \lambda_{j}
$$

This quantity is well defined despite the many identities for the gamma function. The analogue of the Riemannvon Mangoldt formula (for $\zeta$ ) provides the link to the zeros of $\mathcal{L} \in \mathcal{S}^{\sharp}$. If $N(T)$ counts the number of nontrivial zeros $\rho=\beta+\mathrm{i} \gamma$ of $\mathcal{L}$ satisfying $|\gamma|<T$, then

$$
N(T)=\frac{\operatorname{deg} \mathcal{L}}{\pi} T \log T+c T+O(\log T)
$$

with some constant $c$, depending on $\mathcal{L}$, as $T \rightarrow \infty$ (see [34]). The trivial zeros are located at the simple poles of the gamma factors $\Gamma(\lambda s+\mu)$ as they appear on the left hand-side of the functional equation, which are

$$
\rho=-\frac{m+\mu}{\lambda} \quad \text { for } m=0,1,2, \ldots .
$$

It follows that the number of these trivial zeros of absolute value less than $r$ is asymptotically equal to

$$
\sum_{1 \leqslant j \leqslant f} \lambda_{j} r+O(1)=\frac{\operatorname{deg} \mathcal{L}}{2} r+O(1) .
$$

Hence elements of positive degree possess quite a few zeros of both kinds. Moreover, inclusion (1.1) on its own already implies $\operatorname{deg} \mathcal{L}_{1} \leqslant \operatorname{deg} \mathcal{L}_{2}$. 
To get more familiar with this axiomatic setting, let us give some examples. Elements of degree zero in $\mathcal{S}^{\sharp}$ are certain Dirichlet polynomials; the only element of this degree in the subset $\mathcal{S}$ is the function constant 1. Applying ideas of Salomon Bochner [3], Brian Conrey and Amit Ghosh [6] showed that $\mathcal{S}$ contains no any element $\mathcal{L}$ of degree $\operatorname{deg} \mathcal{L} \in(0,1)$, and it is easy to see that their proof carries over to $\mathcal{S}^{\sharp}$; in fact, we will use part of their reasoning in this note too. The degree one elements in $\mathcal{S}$ are the Riemann zeta-function $\zeta$, the Dirichlet $L$-functions $L(s ; \chi)$ associated with a primitive character $\chi$, and their shifts $L(s+\mathrm{i} \theta ; \chi)$ for any real $\theta$.

Notice that already for degree one, there exist examples of elements satisfying essentially the same functional equation, for example, Dirichlet $L$-functions $L(s ; \chi)$ with nonquadratic primitive characters $\chi_{1}, \chi_{2}$ $(\bmod 5)$ satisfying $\chi_{j}(-1)=-1$ and $\chi_{1}=\overline{\chi_{2}}$ and identical trivial zeros.

Jerzy Kaczorowski and Alberto Perelli [12] characterized the elements of degree one in $\mathcal{S}^{\sharp}$ as linear combinations of degree one $L$-functions from $\mathcal{S}$ multiplied by degree zero Dirichlet polynomials. Elements of larger degree are, for example, $L$-functions associated with certain modular forms (degree two) and Dedekind zetafunctions (with degree equal to the dimension of the corresponding number field as a vector space over the rationals). It is conjectured that the Selberg class $\mathcal{S}$ consists of all automorphic $L$-functions and that the degree is always a nonnegative integer. For this and further information, we refer to the survey [13] by Kaczorowski and Perelli and the monograph [26] by Ram Murty and Kumar Murty (resp., [34]).

\section{The beginning of the proof of Theorem 1}

First, we define the quotient

$$
\ell(s):=\frac{\mathcal{L}_{2}(s)}{\mathcal{L}_{1}(s)}
$$

In view of (1.1), every singularity of this function is removable, and hence $\ell$ is analytic throughout the complex plane too. Recall the Dirichlet series representations of the functions $\mathcal{L}_{j}$,

$$
\mathcal{L}_{j}(s)=\sum_{n \geqslant 1} a_{j}(n) n^{-s} \quad \text { for } j=1,2
$$

both absolutely convergent for $\sigma>1$.

Dirichlet series constitute a multiplicative monoid with respect to Dirichlet convolution $*$ of arithmetical functions. Since the constant term $a_{1}(1)$ of $\mathcal{L}_{1}$ is by assumption nonzero, the inverse of its Dirichlet series also exists and converges in some right half-plane; this was first shown by Edmund Landau [16] (see also [1, Sects. 2.7 and 11.9]). Hence, for $d \in \mathbb{Z}$, we may write the corresponding power as

$$
\mathcal{L}_{1}(s)^{d}=\sum_{n \geqslant 1} a_{1}^{(d)}(n) n^{-s}
$$

with appropriate coefficients $a_{1}^{(d)}(n)$ (and $a_{1}^{(1)}(n)=a_{1}(n)$ ) and sufficiently large $\sigma>\sigma_{0}$; this includes the case of negative exponents for the inverse! For nonnegative $d$, we may also write $a_{2}^{(d)}(n)$ for the corresponding coefficients of $\mathcal{L}_{2}^{d}$ (and $a_{2}^{(1)}(n)=a_{2}(n)$ ). Thus

$$
\begin{aligned}
\ell(s) & =\frac{\sum_{k \geqslant 1} a_{2}(k) k^{-s}}{\sum_{\ell \geqslant 1} a_{1}(\ell) \ell^{-s}}=\sum_{k \geqslant 1} a_{2}^{(1)}(k) k^{-s} \cdot \sum_{\ell \geqslant 1} a_{1}^{(-1)}(\ell) \ell^{-s} \\
& =\sum_{n \geqslant 1} \mathfrak{a}(n) n^{-s} \quad \text { with } \mathfrak{a}=a_{2}^{(1)} * a_{1}^{(-1)} .
\end{aligned}
$$


Hence $\ell$ has a Dirichlet series representation in some right half-plane. Next, we will deduce a functional equation for $\ell$.

Rewriting the functional equations for $\mathcal{L}_{1}$ and $\mathcal{L}_{2}$ in an asymmetrical form

$$
\mathcal{L}_{i}(s)=\Delta_{i}(s) \overline{\mathcal{L}_{i}(1-\bar{s})}
$$

for $i=1,2$, where

$$
\Delta_{i}(s):=\omega_{i} Q_{i}^{1-2 s} \cdot \prod_{1 \leqslant j \leqslant f_{i}} \frac{\Gamma\left(\lambda_{j}^{(i)}(1-s)+\overline{\mu_{j}^{(i)}}\right)}{\Gamma\left(\lambda_{j}^{(i)} s+\mu_{j}^{(i)}\right)},
$$

it follows that

$$
\ell(s)=\Delta(s) \overline{\ell(1-\bar{s})} \text { with } \Delta(s):=\frac{\Delta_{2}(s)}{\Delta_{1}(s)} .
$$

We continue with an argument similar to Conrey and Ghosh [6], resp., Bochner [3] (however, we can find traces of this reasoning in the works of Erich Hecke, Hans Hamburger, and even Adolf Hurwitz; see the historical paper [28] by Nicola Oswald).

For this purpose, we consider the Fourier series built with the Dirichlet coefficients $\mathfrak{a}(n)$ of $\ell$ according to (3.2), namely,

$$
f(z):=\sum_{n \geqslant 1} \mathfrak{a}(n) \exp (2 \pi \mathrm{i} n z)
$$

with $z=x+\mathrm{i} y$, which converges in the upper half-plane $y>0$. Using the Mellin inversion (for the gamma function; see Titchmarsh [35, Sect. 9.43]), it follows that

$$
f(z)=\frac{1}{2 \pi \mathrm{i}} \int_{c-\mathrm{i} \infty}^{c+\mathrm{i} \infty} \ell(s) \Gamma(s)(-2 \pi \mathrm{i} z)^{-s} \mathrm{~d} s
$$

where $c>1$ is a constant, and the integration is along the vertical line $\sigma=c$. Next, by shifting the path of integration $c+\mathrm{i} \mathbb{R}$ to $-\infty$ and taking into account the functional equation (3.4) of the entire function $\ell$ with (3.3) and Stirling's formula as well as Friedrich Prym's well-known partial fraction decomposition

$$
\Gamma(s)=\sum_{k \geqslant 0} \frac{(-1)^{k}}{k !} \frac{1}{s+k}+\int_{1}^{\infty} u^{s-1} \exp (-u) \mathrm{d} u
$$

(see [35, p. 162]), it follows from Cauchy's theorem that the remaining residues sum up to

$$
f(z)=\sum_{m \geqslant 0} \frac{\ell(-m)}{m !}(2 \pi \mathrm{i} z)^{m}
$$

if $\mathcal{L}_{2}$ would be allowed to have a pole at $s=1$, then we would have to add here the term $P(\log \mathrm{i} z) / z$, where $P$ is a certain polynomial of degree depending on the order of the pole. Once more using Stirling's formula with (3.3) and the functional equation (3.4) for $\ell$, we have

$$
\frac{\ell(-m)}{m !} \ll C^{m} m^{-(1-d) m} \text { for } m \geqslant m_{0}
$$


with some absolute constant $C>0$ and $d:=\operatorname{deg} \mathcal{L}_{2}-\operatorname{deg} \mathcal{L}_{1}$, which is nonnegative (by (1.1)). This shows that the infinite series in (3.5) defines an entire function if $d<1$; this inequality is indeed satisfied thanks to assumption (1.2). Consequently, $f(z)$ is analytic for $z \in \mathbb{C} \backslash(-\infty, 0]$. However, since $f$ is 1-periodic, it follows that $f$ is entire as well. For $y=\Im z>0$,

$$
\mathfrak{a}(n) \exp (-2 \pi n y)=\int_{0}^{1} f(x+\mathrm{i} y) \exp (-2 \pi \mathrm{i} n x) \mathrm{d} x .
$$

Hence by differentiating $m$-times with respect to $y$ we have

$$
\mathfrak{a}(n) \ll n^{-m} \text { for } m \in \mathbb{N} .
$$

This bound for the coefficients implies that the Dirichlet series representation for $\ell$ converges throughout $\mathbb{C}$ (see [35, Sec. 9.14]), and $\ell$ equals the Dirichlet series $\mathcal{D}$ mentioned in the first statement of the theorem.

If either $\operatorname{deg} \mathcal{L}_{1}=\operatorname{deg} \mathcal{L}_{2}$ or $\mathcal{Z}_{1}=\mathcal{Z}_{2}$, then we can say a little more about $\ell=\mathcal{D}$. In this case, it follows from (2.1) that the gamma factors in $\Delta$ cancel, and hence the functional equation (3.4) takes the form

$$
\sum_{n \geqslant 1} \mathfrak{a}(n) \cdot\left(\frac{Q^{2}}{n}\right)^{s}=\omega Q \sum_{n \geqslant 1} \frac{\overline{\mathfrak{a}(n)}}{n} \cdot n^{s},
$$

where $\omega:=\omega_{2} / \omega_{1}$ and $Q:=Q_{2} / Q_{1}$ (by (3.3) and (3.4)). Taking into account the uniqueness of the Dirichlet series representation (see [35]), it follows that $Q^{2}$ must be integral and $\mathfrak{a}(n)$ is vanishing except for the divisors of $Q^{2}$. Consequently, $\ell$ is a Dirichlet polynomial of the form of the degree zero elements in $\mathcal{S}^{\sharp}$.

Before we continue with statement (ii) in Section 4, we first recall some results from universality theory.

\section{Recapitulating universality}

In 1974/75, Sergei Voronin discovered the following remarkable approximation property of the Riemann zetafunction, called universality: Let $f:\{s \in \mathbb{C}:|s| \leqslant r\} \rightarrow \mathbb{C}$ be a continuous nonvanishing function that is analytic in the interior of the disk of definition, where $0<r<1 / 4$. Then, for every $\epsilon>0$, there exists a real $\tau>0$ such that

$$
\max _{|s| \leqslant r}\left|\zeta\left(s+\frac{3}{4}+\mathrm{i} \tau\right)-f(s)\right|<\epsilon
$$

It can even be shown that for fixed $\epsilon$, the set of these shifts $\tau$ has positive lower density. It is an interesting historical note to mention that the paper [38] was received August 21, 1974 by Izv. Akad. Nauk SSSR, Ser. Mat., which means that the fiftieth anniversary is coming up soon. The very origins of Voronin's spectacular work, however, can be found in Harald Bohr's studies about a century ago.

One of Bohr's insights, formulated in [4], led him to his celebrated theory of almost periodic functions; another consequence was found by Bhaskar Bagchi [2], who deduced an interesting relation to the location of zeros: The Riemann hypothesis is true if and only if the Riemann zeta-function can approximate itself in the following sense: given any disk $D$ in the open right half of the critical strip, the set of $\tau>0$ satisfying

$$
\max _{s \in D}|\zeta(s+\mathrm{i} \tau)-\zeta(s)|<\epsilon
$$

has positive lower density (see also [34, Sec. 8]). In some sense, this note presents another different link between zeros and universality (although the target functions in Voronin's theorem or its generalization to $L$-functions are supposed to be nonvanishing). Our approach is also quite different from the joint paper [8] with Garunkštis and Grahl. 
The aforementioned universality results have been generalized and extended in various ways. For example, Axel Reich [29] proved universality of certain Euler products (including Dedekind zeta-functions); Antanas Laurinčikas and Kohji Matsumoto [19] showed that $L$-functions associated with certain modular forms are universal; universality of polynomial Euler products in the Selberg class (satisfying a prime number theorem) was achieved by Hirofumi Nagoshi and the author [27]; and a universality theorem for the Selberg zetafunction to the full modular group was given by Paulius Drungilas, Ramūnas Garunkštis, Audrius Kačènas [7] (which is of special interest since this function is of order two and therefore off the frame of ordinary Dirichlet series). For further cases like the Lerch zeta-function, we refer to the monograph [18] by Laurinčikas and Garunkštis.

We will return once more to Voronin. In the period 1973-1976, he discovered the following simultaneous approximation phenomenon, called joint universality, for Dirichlet $L$-functions: Given admissible functions $f_{j}$ defined on a disk $|s| \leqslant r$ with $0<r<1 / 4$ and pairwise nonequivalent characters $\chi_{j}$ for $j=1, \ldots, J$, then, for every $\epsilon>0$, there exists $\tau>0$ such that

$$
\max _{1 \leqslant j \leqslant J} \max _{|s| \leqslant r}\left|L\left(s ; \chi_{j}\right)-f_{j}(s)\right|<\epsilon
$$

Again, this set of $\tau$ also has positive lower density for any fixed $\epsilon$. This result can be found in his $\mathrm{PhD}$ thesis [36] from 1977; his paper [37], entitled "On the functional independence of Dirichlet $L$-functions" from 1975, handed in on 12 December 1973, does not explicitly contain joint universality.

This simultaneous approximation also has been extended and generalized. The probably most far-reaching joint universality theorems for zeta- and $L$-functions were obtained by Hidehiko Mishou and Hirofumi Nagoshi [24] and Yoonbok Lee, Takashi Nakamura, and Łukasz Pańkowski [20]; the latter paper contains a proof of a substantial part of a conjecture made by the author [34] (see Section 12.5), namely, that every finite family of primitive $L$-functions within $\mathcal{S}$ should be jointly universal. Indeed, Selberg's yet unproved orthogonality conjecture is expected to provide the necessary independence for this simultaneous approximation property. Lee et al. [20] obtained a joint universality result conditional to a stronger version of this orthogonality conjecture, that is,

$$
\sum_{p \leqslant x} \frac{a_{1}(p) \overline{a_{2}(p)}}{p}= \begin{cases}c \log \log x+\operatorname{error}(x) & \text { if } \mathcal{L}_{1}=\mathcal{L}_{2} \\ \operatorname{error}(x) & \text { otherwise }\end{cases}
$$

where $\mathcal{L}_{j}(s)=\sum_{n} a_{j}(n) n^{-s}$, and $c \neq 0$ is a constant, depending on $\mathcal{L}_{1}$, and the error term is a power series in $(\log x)^{-1}$. Note that the required orthogonality is known for many cases, for example, for automorphic $L$-functions associated with certain irreducible unitary cuspidal representations by Jianya Liu and Yangbo Ye [22]. However, it is not proven so far that every finite family of primitive $L$-functions is jointly universal.

The phenomenon of universality has been intensively investigated in Lithuania, in particular, by Antanas Laurinčikas and Ramūnas Garunkštis and their students (among them, the author :-) . In terms of PhD genealogy, Jonas Kubilius was the father and grandfather, respectively, of them. For an excellent and comprehensive survey on universality theory (including many further faces of ongoing research), we refer to Matsumoto's survey [23]. The first monograph focusing universality is [17] by Laurinčikas, which is still a perfect source for discovering the field; the book [14] of Anatoly Karatsuba and Voronin includes the essential parts of Voronin's thesis.

\section{The end of the proof of Theorem 1}

If we suppose that $\mathcal{L}_{1}$ and $\mathcal{L}_{2}$ are jointly universal (not necessarily with shifts forming a set of positive lower density), then $\ell=\mathcal{L}_{2} / \mathcal{L}_{1}$ is universal too.

Indeed, joint universality for the $\mathcal{L}_{j}$,

$$
\mathcal{L}_{j}(s+\mathrm{i} \tau) \rightarrow f_{j}(s) \text { for } j=1,2,
$$


with admissible functions $f_{j}$, implies universality for their quotient

$$
\ell(s+\mathrm{i} \tau)=\frac{\mathcal{L}_{2}(s+\mathrm{i} \tau)}{\mathcal{L}_{1}(s+\mathrm{i} \tau)} \rightarrow \frac{f_{2}(s)}{f_{1}(s)}
$$

(in fact, this follows proving that the quotient of convergent sequences with nonzero limit converges with limit equal to the quotient of the respective limits). Next, we may simply choose $f_{1} \equiv 1$, proving the uniform approximation property for $\ell$.

This implies the desired contradiction: a Dirichlet series $\ell(s)$ converging in the whole complex plane is bounded for $s$ from the critical strip, and hence it cannot approximate a wide class of analytic functions. It follows that (1.1) or (1.2) cannot hold simultaneously.

Originally, the author had another end of the proof in mind. At that point of research the assumption was $\operatorname{deg} \mathcal{L}_{1}=\operatorname{deg} \mathcal{L}_{2}$, and, consequently, $\ell$ would be a Dirichlet polynomial (as explained at the end of Section 3). However, Dirichlet polynomials satisfy a (linear) algebraic differential equation. This is easily seen by observing in our case that

$$
\ell^{(k)}(s)=\sum_{n \leqslant Q^{2}} \mathfrak{a}(n) \cdot(-\log n)^{k} \cdot n^{-s}
$$

hence

$$
\sum_{0 \leqslant k \leqslant K} x_{k} \cdot \ell^{(k)}(s)=0 \quad \forall s \in \mathbb{C} \quad\left(\exists k: x_{k} \neq 0\right)
$$

iff

$$
\sum_{0 \leqslant k \leqslant K} x_{k} \cdot \mathfrak{a}(n) \cdot(-\log n)^{k}=0 \quad \forall n \leqslant Q^{2}
$$

which has a nontrivial solution if $K>Q^{2}+1$.

On the contrary, universal functions do not satisfy any algebraic differential equation as already Voronin proved (see [36], [34, Sects. 10.1,10.2]). A differential equation restricts the distribution of values drastically!

We could argue a little more generally. In place of (3.1), we could consider

$$
\ell(s):=\frac{\mathcal{L}_{2}(s)^{d_{1}}}{\mathcal{L}_{1}(s)^{d_{2}}} \quad \text { with } d_{j}:=\operatorname{deg} \mathcal{L}_{j}
$$

at least for elements with integral degree, and rewrite (1.1) accordingly; then assumption (1.2) can be dropped. Extending the notion of degree to quotients, it would follow that $\ell$ has degree zero, and hence $\ell$ would be a Dirichlet polynomial, which is, as we have just observed, not compatible with the universality property.

However, with $\mathcal{L}$ also any power is an element of $\mathcal{S}$, and the benefit of this construction is rather limited. More interesting would be to incorporate also $L$-functions having a pole at $s=1$; it is conjectured that any such $\mathcal{L} \in \mathcal{S}$ has the Riemann zeta-function $\zeta$ as a factor in its (unique) factorization into primitive elements.

Another flaw of the above reasoning is the weird assumption to have a nonvanishing coefficient $a_{1}(1)$ to define the reciprocal Dirichlet series. Maybe we could avoid this in explicit examples by applying so-called hybrid universality results incorporating Diophantine conditions on the set of shifts.

\section{Proof of Theorem 2}

Suppose that there are two different factorizations of some element in the (extended) Selberg class into primitive elements, that is,

$$
\prod_{m \in M} \mathcal{L}_{m}^{e_{m}}=\prod_{n \in N} \mathcal{L}_{n}^{e_{n}}
$$


with primitive elements $\mathcal{L}_{j} \in \mathcal{S}$ (or $\mathcal{S}^{\sharp}$ ), without loss of generality finite disjoint subsets $M, N$ of $\mathbb{N}$ (since factors that appear on both sides can be canceled), and exponents $e_{m}, e_{n} \in \mathbb{N}$. Assuming that every finite family of primitive $L$-functions is jointly universal, it follows from approximating appropriate constant functions that, for every $\epsilon>0$, there exists a real $\tau$ such that

$$
\max _{|s| \leqslant 1 / 100}\left|\mathcal{L}_{j}\left(s+\frac{3}{4}+\mathrm{i} \tau\right)-p_{j}\right|<\epsilon \quad \text { for } j \in M \cup N
$$

where $p_{j}$ denotes the $j$ th prime number in ascending order. This implies

$$
\mathcal{L}_{j}\left(s+\frac{3}{4}+\mathrm{i} \tau\right)=p_{j}+O(\epsilon) \quad \text { for } j \in M \cup N
$$

and any fixed $s$ from the disk of approximation or, after substituting this into (6.1),

$$
\prod_{m \in M} p_{m}^{e_{m}}=\prod_{n \in N} p_{n}^{e_{n}}+O(\epsilon)
$$

which contradicts the unique prime factorization of the integers (since by construction every $p_{m}$ on the left is distinct from every $p_{n}$ on the right).

Of course, the reasoning above does not really rely on the (extended) Selberg class; it can be extended to any reasonable multiplicative monoid of $L$-functions (e.g., those associated with other Hecke groups; see [11]). The main question is what the primitive elements are and whether we can prove their joint universality...

\section{Concluding remarks}

Honestly, we have to admit that the key for showing unique factorizations into primitive $L$-functions in $\mathcal{S}$ is probably Selberg's orthogonality conjecture (without the detour via joint universality and Theorem 2). In fact, Ram Murty [26] showed that this orthogonality conjecture implies this unique factorization property in the Selberg class.

A first approach to distinguish $L$-functions is by their Euler products; this reasoning is named strong multiplicity one and applies to $\mathcal{S}$ only. Murty and Murty [25] succeeded to prove that distinct $\mathcal{L}_{1}, \mathcal{L}_{2} \in \mathcal{S}$ have at least constant times $T$ distinct nontrivial zeros $\rho=\beta+\mathrm{i} \gamma$ up to height $T$ in the critical strip. Assuming Selberg's orthogonality relation and a certain density hypothesis, Enrico Bombieri and Alberto Perelli [5] showed for the multiplicities $m_{\mathcal{L}}(\rho)$ of nontrivial zeros $\rho$ that

$$
\sum_{\substack{\rho=\beta+\mathrm{i} \gamma \\ 0<\gamma<T}}\left|m_{\mathcal{L}_{1}}(\rho)-m_{\mathcal{L}_{2}}(\rho)\right| \gg T \log T .
$$

The present best quantitative conditional result had been found by Kannan Soundararajan [32].

The probably strongest statement, however, was obtained by Kotyada Srinivas [33], who proved by a different method unconditionally that

$$
m_{\mathcal{L}_{1}}(\rho)>m_{\mathcal{L}_{2}}(\rho)
$$

for some zero $\rho=\beta+\mathrm{i} \gamma$ of $\mathcal{L}_{1} \cdot \mathcal{L}_{2}$ with $\operatorname{deg} \mathcal{L}_{1} \geqslant \operatorname{deg} \mathcal{L}_{2}$ and $\gamma$ from some neighborhood of $T$ for all sufficiently large $T$. All these papers consider $L$-functions in the Selberg class; it seems that at least in [33] this restriction can be relaxed.

The uniqueness in the extended Selberg class, however, may be a different problem since it is not clear whether a primitive function in $\mathcal{S}$ is necessarily primitive in the larger class $\mathcal{S}^{\sharp}$. 
Acknowledgment. The author is grateful to the valuable comments of the anonymous referee.

\section{Open Access}

This article is licensed under a Creative Commons Attribution 4.0 International License, which permits use, sharing, adaptation, distribution, and reproduction in any medium or format, as long as you give appropriate credit to the original author(s) and the source, provide a link to the Creative Commons license, and indicate if changes were made. The images or other third party material in this article are included in the article's Creative Commons licence, unless indicated otherwise in a credit line to the material. If material is not included in the article's Creative Commons license and your intended use is not permitted by statutory regulation or exceeds the permitted use, you will need to obtain permission directly from the copyright holder. To view a copy of this license, visit http://creativecommons.org/licenses/by/4.0/.

\section{References}

1. T.M. Apostol, Introduction to Analytic Number Theory, Springer, New York, 1976.

2. B. Bagchi, Recurrence in topological dynamics and the Riemann hypothesis, Acta Math. Hung., 50:227-240, 1987.

3. S. Bochner, On Riemann's functional equation with multiple gamma factors, Ann. Math., 67:29-41, 1958.

4. H. Bohr, Über eine quasi-periodische Eigenschaft Dirichletscher Reihen mit Anwendung auf die Dirichletschen L-Funktionen, Math. Ann., 85:115-122, 1922.

5. E. Bombieri and A. Perelli, Distinct zeros of $L$-functions, Acta Arith., 83:271-281, 1998.

6. B. Conrey and A. Ghosh, On the Selberg class of Dirichlet series: Small degrees, Duke Math. J., 72:673-693, 1993.

7. P. Drungilas, R. Garunkštis, and A. Kačènas, Universality of the Selberg zeta-function for the modular group, Forum Math., 25:533-564, 2013.

8. R. Garunkštis, J. Grahl, and J. Steuding, Uniqueness theorems for L-functions, Comment. Math. Univ. St. Pauli, 60: $15-35,2011$.

9. S. Gonek, J. Haan, and H. Ki, A uniqueness theorem for functions in the extended Selberg class, Math. Z., 278:9951004, 2014.

10. E. Hecke, Eine neue Art von Zetafunktionen und ihre Beziehungen zur Verteilung der Primzahlen. I, Math. Z., 1: $357-376,1918$.

11. J. Kaczorowski, G. Molteni, A. Perelli, J. Steuding, and J. Wolfart, Hecke's theory and the Selberg class, Funct. Approximatio, Comment. Math., 35:183-193, 2006.

12. J. Kaczorowski and A. Perelli, On the structure of the Selberg class. I: $0 \leqslant d \leqslant 1$, Acta Math., 2:207-241, 1999.

13. J. Kaczorowski and A. Perelli, The Selberg class: a survey, in K. Győry et al. (Eds.), Number Theory in Progress, Vol. 2, de Gruyter, Berlin, 1999, pp. 953-992.

14. A.A. Karatsuba and S.M. Voronin, The Riemann Zeta-Function, de Gruyter, Berlin, 1992.

15. J. Kubilius, The distribution of Gaussian primes in sectors and contours, Uch. Zap. Leningr. Gos. Univ. Im. A.A. Zhdanova, Ser. Mat. Nauk, Tr. Astron. Obs., 137:40-52, 1950.

16. E. Landau, Über den Wertevorrat von $\zeta(s)$ in der Halbebene $\sigma>1$, Nachr. Ges. Wiss. Göttingen, Math.-Phys. Kl., I(36):81-91, 1933.

17. A. Laurinčikas, Limit Theorems for the Riemann Zeta-Function, Kluwer, Dordrecht, 1996.

18. A. Laurinčikas and R. Garunkštis, The Lerch Zeta-Function, Kluwer, Dordrecht, 2002. 
19. A. Laurinčikas and K. Matsumoto, The universality of zeta-functions attached to certain cusp forms, Acta Arith., 98: 345-359, 2001.

20. Y. Lee, T. Nakamura, and Ł. Pańkowski, Selberg's orthonormality conjecture and joint universality of $L$-functions, Math. Z., 286:1-18, 2017.

21. B.Q. Li, A uniqueness theorem for Dirichlet series satisfying a Riemann-type functional equation, Adv. Math., 226: 4198-4211, 2011.

22. J. Liu and Y. Ye, Perron's formula and the prime number theorem for automorphic $L$-functions, Pure. Appl. Math. Q., 3:481-497, 2007.

23. K. Matsumoto, A survey on the theory of universality for zeta and $L$-functions, in M. Kaneko, S. Kanemitsu, and J. Liu (Eds.), Number Theory: Plowing and Starring Through High Wave Forms. Proceedings of the 7th China-Japan Seminar, Fukuoka, Japan, October 28-November 1, 2013, Ser. Number Theory Appl., Vol. 11, World Scientific, Hackensack, NJ, 2015, pp. 95-144.

24. H. Mishou and H. Nagoshi, The joint universality for pairs of zeta-functions in the Selberg class, Acta Math. Hung., 151:282-327, 2017.

25. M.R. Murty and V.K. Murty, Strong multiplicity one for Selberg's class, C. R. Acad. Sci., Paris, Sér. I, 319:315-320, 1994.

26. M.R. Murty and V.K. Murty, Non-vanishing of L-Functions and Applications, Birkhäuser, Basel, 1997.

27. H. Nagoshi and J. Steuding, Universality for $L$-functions in the Selberg class, Lith. Math. J., 50(3):293-311, 2010.

28. N. Oswald, On a relation between modular functions and Dirichlet series: Found in the estate of Adolf Hurwitz, Arch. Hist. Exact. Sci., 71:345-361, 2017.

29. A. Reich, Universelle Werteverteilung von Eulerprodukten, Nachr. Akad. Wiss. Göttingen, II. Math.-Phys. Kl., K1(1): $1-17,1977$.

30. Z. Rudnick and E. Waxman, Angles of Gaussian primes, Isr. J. Math., 232:159-199, 2019.

31. A. Selberg, Old and new conjectures and results about a class of Dirichlet series, in Collected Papers II, Springer Collect. Works Math., Springer, Berlin, Heidelberg, 1991, pp. 47-64; in E. Bombieri, A. Perelli, and U. Zannier (Eds.), Proceedings of the Amalfi Conference on Analytic Number Theory, Maiori, Italy, 25-29 September, 1989, Università di Salerno, Salerno, 1992, pp. 367-385.

32. K. Soundararajan, Strong multiplicity one for the Selberg class, Can. Math. Bull., 47:464-478, 2004.

33. K. Srinivas, Distinct zeros of functions in the Selberg class, Acta Arith., 103:201-207, 2002.

34. J. Steuding, Value-Distribution of L-Functions, Lect. Notes Math., Vol. 1877, Springer, Berlin, Heidelberg, 2007.

35. E.C. Titchmarsh, The Theory of Functions, 2nd ed., Oxford Univ. Press, Oxford, 1939.

36. S. Voronin, Analytic Properties of Dirichlet Generating Functions of Arithmetic Objects, Thesis Doctor Phys.-Math. Sci., V.A. Steklov Mathematical Institute, Moscow, Russia, 1977 (in Russian).

37. S.M. Voronin, On the functional independence of Dirichlet $L$-functions, Acta Arith., 27:493-503, 1975 (in Russian).

38. S.M. Voronin, Theorem on the "universality" of the Riemann zeta-function, Izv. Akad. Nauk SSSR, Ser. Mat., 39:475486, 1975 (in Russian). 